at a cost of $£ 169$. Mean (SE) costs from hospital discharge to 28 days were $£ 293$ ( $£ 22$ ) per patient. The main cost driver post discharge was readmission to hospital; $12 \%$ of patients were readmitted within 28 days for a mean of 4.8 days. The mean cost associated with readmission across all patients was $£ 127$. HRQoL was on average (SE) $0.68(0.01)$ at 28 days.

Conclusion The mean cost up to 28 days for patients presenting with AUGIB is $£ 2,207$. At 28 days, the mean HRQoL in patients who have experienced an AUGIB is well below the average population level of 0.86 . This is the first study to provide detailed estimates of the costs and HRQoL associated with AUGIB in the UK. These data can be used by healthcare providers and researchers to inform the design of subsequent cost-effectiveness analyses of interventions for AUGIB.

Disclosure of Interest None Declared.

\section{PTU-149 MALNUTRITION AND GASTROINTESTINAL (GI) SYMPTOMS IN PATIENTS WITH UPPER-GI CANCER}

1,2 $\mathrm{E}$ Grace*, ${ }^{3} \mathrm{~K}$ Mohammed, ${ }^{1} \mathrm{C}$ Shaw, ${ }^{2} \mathrm{~K}$ Whelan, ${ }^{3} \mathrm{~J}$ Andreyev. ${ }^{1}$ Department of Nutrition and Dietetics, The Royal Marsden NHS Foundation Trust, UK; ${ }^{2}$ Diabetes and Nutritional Sciences Division, King's College London, UK; ${ }^{3} \mathrm{Gl}$ Unit, The Royal Marsden NHS Foundation Trust, London, UK

\subsection{6/gutjnl-2014-307263.223}

Introduction Persistent GI symptoms and malnutrition have been associated with poorer quality of life in upper-GI cancer patients. This study aims to assess GI symptoms and nutritional status in patients undergoing modern treatment.

Methods Patients with newly diagnosed upper-GI cancer were prospectively reviewed at the time of diagnosis and at 3- and 12months following radical treatment. Nutritional assessment was performed using the patient-generated subjective global assessment (PG-SGA), which is considered the 'gold-standard' for nutritional assessment and has been validated in the oncology setting (score $\geq 4$ intervention needed; score $\geq 9$ critical intervention needed). The gastrointestinal symptom rating scale (GSRS) was used to evaluate the presence/absence and severity of 22 GI symptoms using a 4-point response scale. Total scores range from 0-66, where $0=$ all symptoms absent and $66=$ all symptoms severe.

Results 61 males and 19 females, median age 66 (range 46-89) years were recruited (61\% oesophageal, $33 \%$ gastric, $6 \%$ gastrooesophageal junction tumours). Of these, 68 were reviewed at 3months and 25 at 12-months. Mean (SD) body weight and body mass index (BMI) were $76.7 \mathrm{~kg}(17.4)$ and $26.7 \mathrm{~kg} / \mathrm{m}^{2}$ (4.7) at baseline, $74.4 \mathrm{~kg}(14.8)$ and $25.9 \mathrm{~kg} / \mathrm{m}^{2}(4.4)$ at 3 -months and $72.1 \mathrm{~kg}(16.3)$ and $24.7 \mathrm{~kg} / \mathrm{m}^{2}(4.4)$ at 12 -months. There was a significant mean difference in weight $(-2.0 \mathrm{~kg}, \mathrm{p}=0.002)$ and BMI $\left(-0.56 \mathrm{~kg} / \mathrm{m}^{2}, \mathrm{p}=0.006\right)$ at 3 -months compared to baseline. These reduced further by 12 -months. Mean (SD) PG-SGA score at baseline 9.0 (6.3), 3-months 7.8 (5.6), and 12-months 7.4 (5.0) indicated that intervention was required. At baseline, 3and 12 -months $61 \%, 52 \%$ and $68 \%$ of patients respectively were considered moderately or severely malnourished. Mean (SD) total GSRS scores were 14.2 (10.8), 12.0 (9.4) and 15.5 (11.5) at baseline, 3- and 12-months respectively. The symptoms with the greatest increase in prevalence ( $\%$ more patients) from baseline to 3 -months $(n=68)$ were nausea $(+24 \%)$, loose stool $(+16 \%)$, urgency $(+6 \%)$, flatulence $(+6 \%)$ and early satiety $(+6 \%)$. Those with the greatest decrease in prevalence $(\%$ less patients) during this time were difficulty swallowing (-24\%), painful swallowing (-24\%), regurgitation (-21\%), belching ($15 \%)$ and acid reflux $(-12 \%)$. Of the $n=25$ followed up at $12-$ months, the most common symptoms reported were flatulence (76\%), belching (72\%), abdominal pain (68\%), abdominal grumbling (56\%) and early satiety (52\%).

Conclusion After treatment commences there is progressive weight loss over time. Troublesome GI symptoms persist at 12months and may be contributing to this weight loss. Optimising nutritional status and controlling GI symptoms is required throughout the treatment pathway.

Disclosure of Interest None Declared.

\section{PTU-150 EO: ARE WE GETTING THE MESSAGE YET?}

IA Murray*. Gastroenterology, Royal Cornwall Hospital, Truro, UK

10.1136/gutjnl-2014-307263.224

Introduction Eosinophilic oesophagitis (EO) is the underlying diagnosis in at least $10 \%$ of those with dysphagia. To make the diagnosis, oesophageal biopsies showing an eosinophil count $>15$ per hpf are required. It is most frequent in males under aged 50 years.

Methods All patients having a gastroscopy for dysphagia were identified retrospectively for 6 consecutive years from our endoscopy reporting system. Patient demographics, endoscopic findings and whether biopsies were taken were recorded together with histology results.

Results 3068 patients had a gastroscopy with an indication of dysphagia (1489 female, age 15-100 years, average 67.7 y). The number of patients varied little between years (486-550 patients/year). Common endoscopic diagnoses were normal (20.4\%), benign stricture (12.6\%), oesophagitis (18.1\%), Barrett's (4.8\%), dysmotility (3.7\%) and hiatus hernia (10\%). 1620 (52.8\%) had oesophageal biopsies.

44 patients (1.5\% of all patients) were diagnosed with EO, 32 of who were males. This equates to $2.8 \%$ of those who were biopsied and $4.7 \%$ of those biopsied without cancer, stricture or Barrett's. Although only $13.3 \%$ of those with dysphagia were aged 50 years or under, they equated to $45.4 \%$ of those diagnosed with EO. Of those with EO, 6 had food bolus, 6 "typical" EO changes e.g., feline oesophagus, ridges etc, 4 an irritable oesophagus and 3 Schatzki rings.

Conclusion EO is a relatively common cause of dysphagia but is almost certainly under-recognised due to lack of oesophageal biopsies at endoscopy. Reliance on endoscopic changes of EO at endoscopy will miss the majority of cases. Although biopsying only those under 50 years would be more cost effective than biopsying all, it would also miss the majority of cases. It may be appropriate for the BSG to use frequency of oesophageal biopsies in dysphagic patients as a quality assurance measure for upper GI endoscopy.

Disclosure of Interest None Declared.

\section{PTU-151 PREDICTORS FOR COELIAC DISEASE IN CASES OF LYMPHOCYTIC DUODENOSIS}

${ }^{1}$ A Aziz ${ }^{*},{ }^{2} \mathrm{~T}$ Key, ${ }^{2} \mathrm{JG}$ Goodwin, ${ }^{1} \mathrm{DS}$ Sanders. 'Department of Gastroenterology, Royal Hallamshire Hospital, Sheffield Teaching Hospitals, Sheffield, UK; ${ }^{2}$ Department of Histocompatability and Immunogenetics, Royal Hallamshire Hospital, Sheffield Teaching Hospitals, Sheffield, UK

\subsection{6/gutjnl-2014-307263.225}

Introduction Lymphocytic duodenosis (LD) is an early marker for coeliac disease (CD). However, the majority of cases are due to non-CD related conditions. 